\title{
La perception du dopage : une approche pragmatique
}

\author{
Patrick TRABAL \\ Laboratoire Sport \& Culture, Université Paris X. \\ Émail : ptrabal@u-paris10.fr
}

\begin{abstract}
Résumé - Le but de cet article est d'interroger les méthodes des sociologues cherchant à décrire le dopage sportif. Comme tous les acteurs s'exprimant sur cette question, il nous faut clarifier ce que l'on considère comme "dopage ». Plutôt que de se focaliser sur des définitions statiques, nous préférons mobiliser la sociologie pragmatique en prenant pour objet d'étude les façons dont les acteurs appréhendent la réalité du dopage. Il $s$ 'agit alors de questionner les notions de perceptions, de vigilance et de durée, à partir de deux extraits de confessions de sportifs. On s'aperçoit alors, que la qualification de "dopé », même chez l'intéressé, renvoie à des dimensions sociales complexes.
\end{abstract}

Abstract - The aim of this paper is to poll sociologists'methods with the purpose of describing sports' doping. As any actor working on this subject, we need to clarify what "doping" is. Instead of focusing on static definitions, we prefer with pragmatic sociology and are studying the ways used by actors to grasp the reality of doping. So, we need to question notions like perception, vigilance and duration, from two excerpts of sportsmen' confessions. We then notice that the labelling of « doped ", even for the person concerned, refers to complex social aspects.

Mots clés-Dopage-Sociologie-Méthode-Perception-Temporalité. 


\section{Introduction}

La question du dopage, comme toute pratique déviante impliquant le secret (Becker, 1985), pose aux sciences sociales d'importantes difficultés dont les fondements théoriques, épistémologiques et pratiques constituent un véritable défi à nos disciplines. Pour s'en convaincre, il suffit de recenser la faiblesse des méthodes habituelles mobilisées par le sociologue pour appréhender des pratiques sociales. Classiquement, on recense quatre grands types d'outils : le questionnaire, l'entretien, l'observation et l'étude des traces (Ghiglione et Matalon, 1991).

Le recours au questionnaire pour quantifier les pratiques ou les conduites dopantes est fréquent chez certains auteurs travaillant sur le dopage et s'inscrit dans une préoccupation épidémiologique. De nombreux sociologues ont pointé les problèmes liés à ce « recueil de données ». Cette expression suggère qu'il suffit de rassembler un « déjà-là » alors que, précisément, les « données » ne sont pas particulièrement « données » puisqu'elles résultent d'un travail sociologique, fondé sur un dispositif de contrôle (le propos des individus est « recueilli » dans le but d'être codé dans les cases du questionnaire) et sur une mise en équivalence. Indépendamment de la qualité des questionnaires mobilisés et des catégories fixées par le chercheur, il demeure une "tension », particulièrement saillante dans la question du dopage, « entre les exigences de la qualification qui précède tout classement et les résistances de la matière à classer » (Boltanski et Thévenot, 1991, p. 12). En d'autres termes, l'opération par laquelle on associe une conduite, une consommation ou une pratique à une catégorie («addicte», « consommateur», « dopé »...) n'est pas neutre. Aussi le résultat de ce travail peut-il difficilement être pris comme des « données ».

Les autres outils du sociologue présentent eux aussi des difficultés. L'observation, notamment, est impossible si l'on ne dispose pas d'une position particulière permettant d'être témoin des pratiques à analyser. L'étude des traces, c'est-à-dire la recension d'éléments laissés par les acteurs (par exemple dans les poubelles), comme dans une certaine mesure, l'entretien, renvoient à un autre problème : en quoi l'enquête du sociologue se distingue-t-elle de celle des policiers, ou de celle des médecins chargés de la lutte antidopage ? Insister sur le fait que le chercheur s'emploie à comprendre lorsque les autres visent à sanctionner ne suffit guère. Comment, en effet, affirmer des différences entre les scientifiques lorsque ceux-ci, partagent les mêmes titres ("Docteur», 
« Professeur ») et les mêmes objectifs (lutter contre le dopage ${ }^{1}$ ) ? En fait, cette activité est aussi partagée par d'autres acteurs, notamment par les journalistes, qui eux aussi, tentent d'appréhender la question du dopage. Ainsi, ce sont bien tous les acteurs qui souhaiteraient connaître la réalité des pratiques dopantes. Les finalités attachées à cet objectif varient. Les sportifs, leurs entraîneurs et leurs équipes médicales aimeraient connaître les pratiques de leurs concurrents. La plupart des responsables politiques et sportifs, alliés de scientifiques et médecins, visent à mieux appréhender le phénomène pour l'éradiquer. Les personnes chargées des contrôles antidopage, les policiers et les juges s'emploient à débusquer les contrevenants afin de faire respecter les règles. Les journalistes essaient de démasquer les tricheurs pour informer les spectateurs et valoriser le spectacle sportif (Duret et Trabal, 2001). Le sociologue, lui aussi, tente de connaître et de comprendre ces pratiques, pour faire avancer la connaissance et, parfois, pour participer, à son niveau, à la lutte antidopage.

\section{La pragmatique}

La question sur laquelle buttent donc tous les acteurs est bien celle-ci : quelle est la réalité du dopage ? Derrière cette interrogation, en figure une autre : quelle est la spécificité du travail du sociologue, si au bout du compte, d'autres acteurs (médecins, journalistes...) se posent la même question et semblent mobiliser des outils semblables (questionnaires, étude de traces, entretiens...).

Une voie de sortie de ces questions est peut-être la sociologie pragmatique qui se propose de prendre pour objet ces interrogations. En effet, selon William James, un des pères du pragmatisme, une des voies pour comprendre les actions des individus consiste à se demander « dans quelles conditions ils considèrent que les choses sont réelles ». Dans cette perspective, l'on peut tenter de recenser et de retracer leurs médiations des différents acteurs lorsqu'ils considèrent le dopage. En particulier, on peut essayer de repérer les éléments mobilisés pour rentrer dans l'arène, éléments qui peuvent eux aussi faire l'objet de débat.

Envisageons le cas des sportifs. Leur appréhension avec le dopage se traduit essentiellement par leur corps. Cela peut être directement par un contact physique entre un produit « injecté », « absorbé » ou « déposé » sur le corps, dans le cas des sportifs dopés. Leur rapport au dopage peut aussi passer par leur

1 Certes, généralement, le sociologue ne considère pas la lutte contre le dopage comme un élément prioritaire de son travail. Mais cet aspect ne lui est pas étranger puisque, le plus souvent, il est lié à un appel d'offre (s'inscrivant dans la lutte antidopage) ou à un engagement personnel pour cette question (que ce soit au nom de l'éthique sportive ou de questions sanitaires). 
corps « témoignant» (Dulong, $1998:$ 192-196) lorsque, par exemple, leurs collègues, se livrent ouvertement à ces pratiques. Un entraîneur peut parler du dopage s'il repère, en un coup d'œil (encore une partie du corps, mais qui cette fois fonctionne à distance) quelqu'un qui est «chargé $»^{2}$. En revanche, la personne se livrant à un contrôle n'accédera à la réalité du dopage que par un ensemble de dispositifs. L'on perçoit ici que les médiations sont plus longues. B. Latour (par exemple, 1989) invite à les décrire en retraçant tous les maillons de la chaîne, reliant ici le sportif et l'observateur, et à repérer les plus faibles d'entre eux. En effet, la fragilité d'un élément de la chaîne devient celle de l'ensemble du réseau. Il suffit, par exemple, de souligner comment un doute dans la méthode française de détection de l'EPO peut ébranler l'ensemble de l'édifice ${ }^{3}$. Considérons un dernier acteur : le responsable politique. Son accès au dopage passe par des dispositifs comme les instruments des personnes chargées des contrôles antidopage. Ne pouvant cependant accéder directement à une lecture des différentes mesures, il lui faut des médiations supplémentaires : des rapports des responsables des tests et des statistiques. Or des chercheurs comme A. Desrosières (1993) ont remarquablement décrit les forces et les faiblesses des appareils statistiques, qui fonctionnent simultanément par une totalisation et une réduction de la réalité. Cette brève analyse des modes d'accès au dopage, qui passent par des corps, des dispositifs, des configurations, suggère la nécessité de poursuivre systématiquement un tel travail en recensant tous les acteurs et la variété des médiations engagées. La tradition pragmatique invite à penser que celles-ci sont directement liées à la diversité des positions dans les débats et donc aux ressorts de la controverse. Cette perspective semble à la fois classique en sociologie mais elle impose de partir des contraintes des acteurs et des médiations qu'elles leur permettent de mobiliser; du coup, elle est en fait en rupture avec des approches posant les positions sociales comme prédominantes.

Cet article se propose d'expliciter notre travail (qui ne fait que commencer), en montrant comment la pragmatique aborde la notion de perception, largement utilisée pour évoquer les pratiques qui nous intéressent.

2 Willy Voet raconte dans son livre comment il peut " reconnaître huit fois sur dix, à des petits signes imperceptibles pour d'autres, qui est "chargé" et qui ne l'est pas " (Voet, $1999: 10)$.

3 Cf. par exemple, Le Monde du 30 novembre 2001. 


\section{Être « dopé » (ou être « toxicomane »)}

Ce programme revient donc à s'attarder sur la façon dont les acteurs considèrent que telle ou telle pratique relève du dopage ou de la toxicomanie. On pourrait considérer que cette qualification ne pose pas de problème dans le cas des sportifs eux-mêmes. Pourtant, des confessions récemment publiées par des anciens dopés tendent à infirmer cette évidence. Jérôme Chiotti, vététiste repenti, raconte dans son livre son premier contrôle antidopage :

«Un petit vent de panique s'empara de moi, même si deux ou trois coureurs m'avaient expliqué que les corticoïdes étaient indétectables et que je ne risquais absolument rien. Je fus vite soulagé. N'étant pas "contrôlable", je ne me considérais nullement comme un hors-la-loi. » (Chiotti, p. 37).

De même, Philippe Boyer, un des témoins se livrant à Yves Bordenave, raconte :

«Toujours est-il qu'une fois le vélo raccroché au clou, les amphés ne m'abandonnent pas. Au contraire. Entre 1992 à 1994, il ne passe pas une semaine sans que j'y touche. Parfois, c'est encore plus resserré. Je me donne bonne conscience. Je m'allume (expression en usage pour la prise d'amphétamine, NDLR) et je vais rouler aussitôt après. Je me dis que je contrôle, que je ne suis pas dépendant. (...) Je n'ai pas conscience d'être un toxicomane». (Bordenave et Simon, p. 94).

Que dire de ces deux témoignages ? Certes, l'on peut considérer que sous l'apparente sincérité de l'individu se confessant, réside un fond de mauvaise foi. Comment imaginer qu'un consommateur de corticoïdes se présentant à un contrôle antidopage parvienne à se persuader qu'il ne transgresse pas la règle ? Ce type d'interrogation conduit à retranscrire le récit de ces champions déchus en terme d'intérêt et de stratégie. Une telle présentation de soi, mariant le regret et la bonne foi, peut-elle être ramenée à une question d'intérêt ? Si on accepte que l'on puisse avoir « intérêt au désintéressement » (Bourdieu, 1997, p. 148), il convient de partir des intérêts et des positions des différents acteurs. Chiotti, dans ces conditions, utiliserait une stratégie bien connue : «j'ai fauté mais je n'en avais pas conscience ». Cette lecture trouve un appui dans le premier texte français de loi antidopage de 1965, puisqu'il faut avoir agi « sciemment » pour être en infraction à la loi. Ainsi, cette déclaration de Chiotti, lui permettrait-elle de reconnaître ses torts en se disculpant, « Responsable mais pas coupable ». La formule n'est pas nouvelle. La loi de 1965 n'est plus en vigueur, mais pourquoi ne pas considérer que les stratégies qu'elle a engendrées, demeurent comme des points d'appuis des fautifs ? La seconde citation, celle de Philippe Boyer, résiste 
moins à cette lecture en termes d'intérêt et de stratégie. Si l'on peut admettre que l'addiction est liée à une mauvaise impression de la capacité à contrôler sa consommation, on peut aussi considérer que le témoignage participe d'une stratégie de reconquête du public. Affirmer que l'on ne voyait pas l'évidence reviendrait là encore à avouer une faute sans accepter les sanctions correspondantes.

À nos yeux, il semble pourtant bien difficile de réduire, coûte que coûte, ces récits à une stratégie. Renoncer à cette lecture ne signifie d'ailleurs pas que l'on renonce à considérer les intérêts des acteurs sociaux. Il s'agit en revanche de ne pas les déposséder de valeurs morales. À la suite des sociologues qui ont renoué avec l'approche pragmatique en étudiant l'importance des considérations morales dans les actions des individus (par exemple : Boltanski et al., 1984 ; Boltanski, 1990 ; Boltanski et Thévenot, 1991 ; Chateauraynaud, 1991 ; Dodier, 1993 ; Chateauraynaud et Torny, 1999 ; Lemieux, 2000), nous considérons que l'on doit prendre au sérieux leurs sens moral et politique. Dans ces conditions, si on renonce à ne lire que de l'intérêt dans les deux extraits cités précédemment, on peut tenter de comprendre comment la qualification de « dopé » s'impose aux acteurs. La question de William James, invitant à interroger les médiations par lesquelles on considère que les choses sont réelles, prend alors tout son sens.

En effet, il semble intéressant dans cette perspective, de décrire les épreuves à l'issue desquelles Chiotti, Boyer et les autres finissent par accepter des qualificatifs comme «dopé » ou «toxicomane». Les confessions des champions repentis font apparaitre que l'on peut consommer des produits interdits sans pour autant accepter d'être un sportif dopé. Cela nous conduit à étudier la perception du dopage des sportifs.

\section{Une sociologie de la perception et du temps}

Souvent le mot « perception » est utilisé pour le mot « représentation », qui est aussi assimilé à « image » ou « conception ». Notre approche invite à clarifier ces notions. Dans leur travail sur l'expert devant « juger » l'authenticité d'un objet d'art, C. Bessy et F. Chateauraynaud, proposent une « sociologie de la perception » qui tente de mettre à distance les usages courants de ces concepts :

« Faut-il opposer un niveau de perceptions claires et distinctes, auquel aurait accès le véritable expert, à un niveau de représentations et d'interprétations socialement variables ? Les perceptions seraientelles « objectives » et les interprétations « subjectives » ? À moins que ce ne soit l'inverse : les perceptions seraient livrées à l'arbitraire des expériences, et les interprétations seraient fondées sur des conventions 
stabilisées ? Ou plutôt ceci : seuls les instruments seraient objectifs, parce qu'en rapport étroit avec le monde physique, et les perceptions et représentations seraient socialement variables et donc quelque peu arbitraires! Mais les instruments ne sont-ils pas aussi des constructions sociales? (C. Bessy et F. Chateauraynaud, 1995, p. 234).

L'idée est de construire la « prise » comme reliant d'une part des repères, notamment grâce à des qualifications qui renvoient tant à des concepts (langages de description, des espaces de calculs, représentations) qu'à des réseaux et, d'autre part, à des « plis » (Deleuze, 1988) c'est-à-dire des « corps-à-corps » engageant non seulement des dispositifs sensoriels, des perceptions, des états du corps, mais aussi des instrumentations (Ibid., p. 243).

Explicitons ces notions en prenant l'exemple du sportif consommateur de produits illicites. D'une part, la «prise » de cette substance, qu'elle se déroule par une injection, une absorption ou une application sur la peau, engage manifestement le corps. Il s'agit à ce titre d'un « corps-à-corps », au sens propre, avec le produit. Des instruments, par exemple une seringue, peuvent jouer un rôle central dans cette « prise ». Or, une telle pratique ne peut être dissociée d'un ensemble de repères constitués par des espaces de calcul et des langages de description (notre sportif sait ce que sont les amphétamines, les anabolisants, l'EPO...) mais aussi par des réseaux (le peloton, l'ensemble des produits disponibles, le circuit d'approvisionnement...). La «prise» d'une substance émerge alors de la rencontre entre tous ces éléments : prendre une ampoule d'un produit dont on connait la nature et les effets, la casser, en verser une quantité donnée dans une seringue, préparer une partie de son corps à recevoir l'aiguille, presser sur le piston, nettoyer et dissimuler l'impact sur le corps mais aussi les traces de cette pratique (en cachant par exemple le matériel, mais aussi en employant des procédés la rendant indétectable).

Cependant, le consommateur d'un produit dopant peut par ailleurs ne pas se considérer forcément comme « dopé ». Pourquoi ? Comment est-ce possible ? Si la «prise» de produit naît de la rencontre entre des repères (parmi lesquels figure notamment la liste des interdits) et les «plis », comment peuton penser les propos de Philippe Boyer ou de Jérôme Chiotti ?

Pour tenter de comprendre cette situation, nous suivrons deux voies, qui en fait, sont liées. La première revient à interroger les repères de l'individu. On peut en effet imaginer qu'ils ont disparu, ou plus exactement, qu'ils se sont déplacés. Avec l'habitude de transgresser dans certaines situations, une limite, un autre seuil s'instaure. Ce processus fonctionne comme la répétition de dépassements ponctuels de la vitesse autorisée sur autoroute qui, dans chacun des cas n'apparaît pas au conducteur comme une infraction: la vitesse que l'on 
s'autorise occasionnellement et dans certaines conditions devient la vitesse de croisière. Seuls les dispositifs mobilisés par les forces de l'ordre et les sanctions qui les accompagnent constituent les repères stables. « N'étant pas "contrôlable", je ne me considérais nullement comme un hors-la-loi » affirme Chiotti. Le modèle de la « prise » proposée par C. Bessy et $\mathrm{F}$. Chateauraynaud suggère que les repères sont constitués de différents éléments suffisamment stabilisés pour fixer les descriptions du monde, par exemple, le métabolisme, les effets des anabolisants, les listes des institutions sportives stipulant leur interdiction dans les courses. On peut donc considérer que l'un des repères (les interdictions) a été déplacé : « est dopé » celui qui est contrôlé positif (repère fixé par un tiers).

L'autre voie invite à étudier le temps. Analyser ce déplacement des repères suppose une prise en compte de la dimension temporelle. Que signifie en effet « être dopé » (ou « être toxicomane », ou au contraire « ne pas l'être ») ? Une telle affirmation suppose que la situation est suffisamment stable pour pouvoir asseoir un jugement. Or il existe une tension entre cette apparente stabilité, sur laquelle fonctionne l'habitude (les « prises » de produits, comme les contrôles antidopage, se succèdent sans que cela ne constitue des événements marquants), et d'autre part, ce changement à l'issue duquel les repères se sont finalement déplacés. Ainsi, le consommateur de produits dopants refusant de se considérer comme «dopé », est-il pris dans un jugement s'appuyant sur une situation antérieure (par exemple, lors de la première expérience dans un contexte bien particulier), sans qu'il lui apparaisse nécessaire de réviser la validité et l'actualité de ce jugement.

Il reste à décrire la façon dont le jugement peut fonctionner sans évaluer la réalité des repères sur lesquels la « prise » s'exerce. En fait, il est sans doute plus facile de travailler à l'envers, c'est-à-dire de décrire les conditions permettant de s'assurer de son jugement : on comprendra alors ce qui peut conduire à l'oubli de l'évaluation des repères.

À la suite de C. Bessy et F. Chateauraynaud, on peut considérer qu'une prise est réussie lorsque les représentations, les sensations corporelles, les mesures des instruments évaluant les états biologiques, chimiques et physiques sont en adéquation. L'on peut en effet se satisfaire de son jugement après avoir confronté ces différents aspects (dans notre cas, la liste des produits dopants, les usages et les dires des autres sportifs, les impressions physiques, l'aiguille de la centrifugeuse, le verdict du laboratoire antidopage...). Le moindre écart peut être le signe invitant à réviser son jugement. Il devient donc particulièrement important de le mettre régulièrement à l'épreuve puisque, dans notre perspective, la perception fonctionne sur la sensibilité à la variation. Saisir le moindre indice suppose en effet un renouvellement régulier du jugement (entendu dans le sens précédent d'une «prise » d'indicateurs informant de la réalité). 
On peut donc penser que le sportif, consommateur régulier d'amphétamines, ne se considérant pas comme toxicomane ou dopé, n'a pas suffisamment renouvelé son jugement en le mettant à l'épreuve. Il s'appuie sur des repères trop anciens, c'est-à-dire non remis à l'épreuve récemment. Il n'a pas été suffisamment vigilant, pour reprendre le terme du philosophe R. Duval (1991), faisant de cette notion un élément central pour penser le temps.

Cette hypothèse est séduisante pour expliquer les défauts de « prise » mais ne suffit pas. Elle ne rend pas très bien compte de l'attitude des sportifs qui généralement attachent une grande importance à leurs sensations corporelles pour évaluer leur état. Les médecins du sport que nous avons rencontrés soulignent combien leurs patients sont attentifs à la moindre anomalie concernant leur état physique. Comment, dans ces conditions, imaginer un manque de vigilance des sportifs, notamment lorsque ceux-ci ne cessent de s'inquiéter de leur forme physique à chaque entraînement et à chaque compétition? La performance du geste sportif dont se nourrissent les athlètes constitue en effet un repère décisif dans le jugement. L'apparition d'une douleur comme la marque indiquée par les métrologies enregistrant les exploits (notamment dans les sports où l'épreuve est jugée « objectivement » par des instruments) figurent en effet comme des appuis pour le jugement du sportif sur sa condition physique.

On peut penser, en revanche, que les indices sont mal interprétés. Repérer une sensation particulière ne suffit pas. On peut en effet la relier à d'autres causes. Pour s'assurer de cette " prise », il faut inscrire cette variation dans une série pour lui donner un sens. Ainsi, il nous semble particulièrement heuristique d'étudier la façon dont les sportifs (mais plus généralement les différents acteurs sociaux) mobilisent des précédents pour juger des situations. Ceux-ci constituent en effet d'autres repères. Une douleur (ou au contraire une sensation de bonne forme) n'a de sens que si elle est replacée dans un espace de variation dans lequel est inscrit un ensemble de sensations vécues par le sportif. Or la répétition de ses sensations (notamment par la régularité des entraînements et des compétitions) rend plus difficile la sensibilité à la variation dans cette série. À la suite de G. Deleuze (1968), F. Chateauraynaud et D. Torny développent cette notion de « série » comme une « accumulation de petites différences à travers la répétition qui finissent par trouver la raison de leur progression, leur loi de transformation leur finalisation » (Chateauraynaud et Torny, 1999, p. 88). Le modèle proposé par les auteurs vise à étudier les conditions sociales nécessaires pour qu'une alerte sanitaire soit prise en compte. Il nous semble intéressant de le transposer non seulement à la question du dopage entendue comme la somme des débats sur ce sujet, mais aussi à la perception du sportif, qui devant une série de signes, prend ou non, la mesure de son état afin de statuer. 
Cette piste suggère donc de s'attarder aux conditions dans lesquelles des indicateurs sont rassemblés pour s'inscrire dans une série ou au contraire pour rester comme des marques isolées, en d'autres termes pour s'imposer, ou non, comme des éléments pertinents à intégrer dans la perception du dopage.

\section{Conclusion}

Le but de cet article était essentiellement de décrire un programme de recherches. Ce dernier est mis en œuvre au sein du séminaire «dopage et sciences sociales » rattaché au laboratoire Sport \& Culture de l'Université Paris-X-Nanterre. Il ne fait que commencer mais nous espérons cependant avoir montré les perspectives ouvertes.

Nous avons tenté de montrer la voie proposée par l'approche pragmatique notamment lorsqu'il s'agit d'étudier la façon dont les acteurs appréhendent la réalité du dopage. Pour souligner la pertinence et la difficulté de cette question, nous nous sommes contentés d'étudier le cas du sportif qui bien que consommateur de produits refuse la qualification de « dopé ». Il ne s'agit pas d'affirmer que cette situation est courante car nous ne sommes pas dans une perspective épidémiologique. Notre propos était simplement d'envisager un cas limite, dans lequel la qualification de «dopé » ne semblait pas poser de problème, et qui pourtant, livre après analyse, sa complexité et son intérêt.

Si l'on travaille systématiquement sur des récits de sportifs, nous pourrons mettre en variation des situations où les questions de réalité et de perception du dopage sont en jeu. Il s'agira ensuite d'étendre notre investigation aux autres acteurs afin d'analyser la dynamique des débats. À nos yeux, c'est au prix de ce long travail, qui pour l'essentiel reste à accomplir, que l'on pourra analyser les controverses et les polémiques sans les réduire à une lecture, qui en partant des positions sociales des agents, oublie de «suivre les acteurs » au plus près de leurs préoccupations. Notre propos consiste à prendre à la lettre cette formule, rassemblant les chercheurs sous la bannière des «nouvelles sociologies » (Corcuff, 1995) en étudiant la façon dont se construisent les perceptions du dopage.

\section{Bibliographie}

BECKER H. S., Outsiders, Première édition en anglais en 1963, Paris, A. M. Métailié, 1995. BESSY C., CHATEAURAYNAUD F., Experts et faussaires, Paris, A. M. Métailié, 1995. BOLTANSKI L., L'amour et la justice comme compétences, Paris, A. M. Métailié, 1990. 
BOLTANSKIL., DARRÉ Y., SCHILTZ M. À, La dénonciation, Actes de la recherche en sciences sociales, 1984, 51, 3-40.

BOLTANSKI L., THÉVENOT L., De la justification, Paris, Gallimard, 1991.

BORDENAVE Y., SIMON S., Paroles de dopés, Paris, J. C. Lattès, 2000.

BOURDIEU P., Méditations pascaliennes, Paris, Seuil, 1997.

CHATEAURAYNAUD F., La faute professionnelle : une sociologie des conflits de responsabilité, Paris, A. M. Métailié, 1991.

CHATEAURAYNAUD F., TORNY D., Les sombres précurseurs - une sociologie pragmatique de l' alerte et du risque, Paris, Éditions de l'École des Hautes Études en Sciences sociales, 1999.

CHIOTTI J., De mon plein gré, Paris, Calmann-Lévy, 2001.

CORCUFF P., Les nouvelles sociologies, Paris, Nathan, 1995.

DELEUZE G., Différence et répétition, Paris, PUF, 1968.

DELEUZE G., Le pli - Leibniz et le baroque, Paris, Minuit, 1988.

DODIER N., 1993, L'expertise médicale - Essai de sociologie sur l'exercice du jugement, Paris, A. M. Métailié.

DULONG R., Le témoin oculaire : les conditions sociales de l'attestation personnelle, Paris, Éditions de l'École des Hautes Études en Sciences sociales, 1998.

DURET P., TRABAL P., Le sport et ses affaires - Une sociologie de la justice de l'épreuve sportive, Paris, A. M. Métailié, 2001.

DUVAL R., Temps et Vigilance, Paris, Vrin, 1991.

GHIGLIONE R., MATALON B., Les enquêtes sociologiques : théories et pratique, Paris, Armand Colin, 1991.

LATOUR B., La science en action, Première édition en anglais en 1987, Paris, La Découverte, 1989.

LEMIEUX C., Mauvaise Presse, Paris, A. M. Métailié, 2000. 